
werden wichtige Arbeiten aus der internationalen Fachliteratur referiert und kommentiert.

\section{Azelastin-Fluticason-Kombination lindert allergische Rhinitis}

\begin{abstract}
Mit einer Kombination des Antihistaminikums Azelastin und des Glukokortikoids Fluticason in einer intranasal zu verabreichenden Präparation zeichnet sich eine neue potente Therapieoption bei allergischer Rhinitis ab.
\end{abstract}

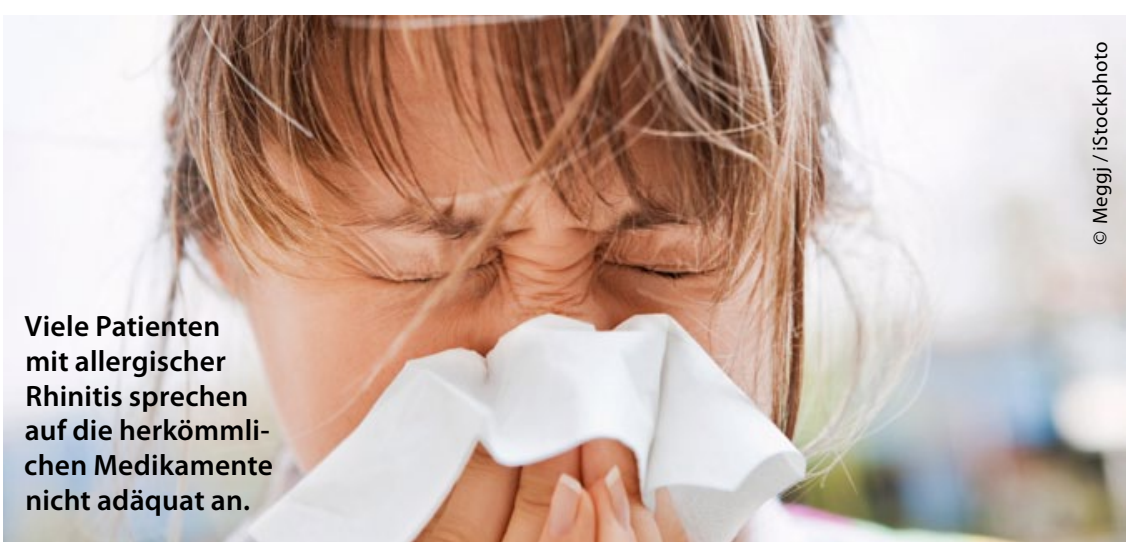

n einer Reihe von Studien hat das intranasale Azelastin-Fluticason-Kombinationspräparat mit der Bezeichnung MP29-02 seine therapeutische Potenz bei Patienten mit allergischer Rhinitis belegen können. Über einige der Resultate wurde bereits auf dem Jahrestreffen der American Academy of Allergy, Asthma, and Immunology (AAAAI) in Orlando, Florida, berichtet. Dabei zeigte sich zum einen eine gute Verträglichkeit auch bei einjähriger Dauertherapie [1]. Zum anderen war die Kombinationstherapie, die Linderung nasaler und okulärer Symptome betreffend, der Einzelgabe der Substanzen bzw. einer Placebobehandlung überlegen [2]. MP29-02, das auch unter der vorläufigen Bezeichnung „Dymista“ firmiert, wurde hierfür jeweils in einer Gesamtdosis von $548 \mu \mathrm{g}$ Azelastin und $200 \mu \mathrm{g}$ Fluticason verabreicht - verteilt auf einen Sprühstoß pro Nasenloch zweimal täglich.
Publiziert wurden auch die Resultate dreier randomisierter und doppelt verblindeter Multizenterstudien sowie einer Metaanalyse hierzu, in denen wiederum die Kombination MP29-02 gegen Azelastin bzw. Fluticason allein und gegen Placebo getestet wurde [3]. Beteiligt waren 3.398 Patienten. Wesentliches Messinstrument war der totale nasale Symptomscore (TNSS), mit dem auf einer Skala von 0 (keine Symptome) bis 24 (schwere Symptome) der Grad der nasalen Obstruktion, des Juckens, des Niesens und der Rhinorrhö bewertet wird. Der Ausgangswert lag dabei im Mittel bei 18 Punkten. 14-tägige MP29-02-Behandlung verbesserte den Wert durchschnittlich um 5,7 Punkte. Unter Fluticason betrug die Senkung 5,1, mit Azelastin 4,4 Punkte. Placebo verbesserte den Score um 3 Punkte. Die Wirkung von MP29-02 war vom ersten Tag an spürbar. Eine 50\%ige Reduktion des TNSS-Wertes wurde unter MP29-02 drei
Tage früher als unter Fluticason und fünf Tage früher als unter Azelastin erreicht.

Im sekundären Endpunkt wurde der okuläre Symptomscore (TOSS) ermittelt, der von 0 bis 18 reicht und mit dem Jucken, Wässrigkeit und Rötung der Augen beurteilt werden. Der Ausgangswert lag hier bei 12 Punkten, MP29-02 verbesserte den Score um 3,2 Punkte. Das war mehr als unter Azelastin $(2,9)$ und Fluticason $(2,8)$ allein bzw. unter Placebo $(1,8)$.

In einer weiteren, ähnlich angelegten Untersuchung mit 779 Patienten mit mäßig bis schwer ausgeprägter saisonaler all-ergischer Rhinitis ließen sich diese positiven Ergebnisse im Wesentlichen bestätigen [4].

Fazit: Viele Patienten, die an allergischer Rhinitis leiden, sprechen nicht adäquat auf die verfügbaren Medikamente an. Oft wird die Schwere der Erkrankung unterschätzt, und es kommt trotz der Therapie zu heftigen Beschwerden. Das führt zu Frustration und mangelnder Compliance. Mit MP29-02, einer Kombination aus Azelastin und Fluticason in einem Nasenspray, lassen sich diese Probleme womöglich vermeiden. In den bisherigen Studien zeigte sich das Präparat zuverlässig wirksam und den Einzelsubstanzen überlegen. Überdies war es gut verträglich. $r b$

1. Berger WE et al. Long-term safety study of MP29-02 in subjects with chronic allergic or non-allergic rhinitis. Meeting of the AAAAI 2012; Abstract 508

2. Rantner P et al. MP29-02 in the treatment of ocular symptoms of seasonal allergic rhinitis. Meeting of the AAAAI 2012; Abstract 509 3. Carr W et al. A novel intranasal therapy of azelastine with fluticasone for the treatment of allergic rhinitis. J Allergy Clin Immunol 2012; 129: 1282-9 4. Meltzer EO et al. MP29-02 in the treatment of seasonal allergic rhinitis: a randomized, doubleblind, placebo-controlled trial of efficacy and safety. Allergy Asthma Proc 2012; 33: 324-32 